Musées, Patrimoine et Culture scientifiques et techniques

$158 \mid 2015$

mars-avril 2015

\title{
Les collections extra-européennes : 25 ans après
}

\section{Roger Boulay}

URL : http://journals.openedition.org/ocim/1502

DOI : 10.4000/ocim.1502

ISSN : 2108-646X

\section{Éditeur}

OCIM

Édition imprimée

Date de publication : 1 mars 2015

Pagination : 31-34

ISSN : 0994-1908

Référence électronique

Roger Boulay, « Les collections extra-européennes : 25 ans après », La Lettre de I'OCIM [En ligne], 158 | 2015, mis en ligne le 01 mars 2016, consulté le 30 avril 2019. URL : http:// journals.openedition.org/ocim/1502; DOI : 10.4000/ocim.1502

Ce document a été généré automatiquement le 30 avril 2019.

Tous droits réservés 


\title{
Les collections extra-européennes : 25 ans après
}

\author{
Roger Boulay
}

L'article (disponible sur www.ocim.fr, rubrique La Lettre de l'OCIM) était en partie consacré au travail d'inventaire des collections d'objets océaniens entrepris par le musée des Beaux-Arts de Boulogne-sur-Mer.

\section{OCS}

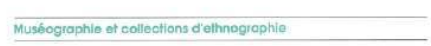

Actualité d'un débat qui s'éternise :

Que foire des collections d'objels exotiques

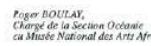

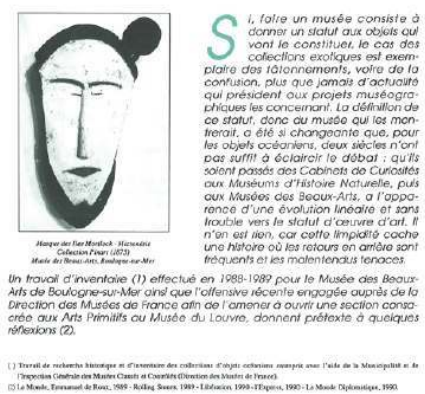

1 En 1990 la situation des collections extra européennes en France s'énonçait en quelques lignes : 
2 - abandon de collections importantes comme celle de Lille alors entreposée dans les caves du musée des Beaux-Arts ou celle de Boulogne-sur-Mer entassée depuis la fin de la guerre dans un grenier ouvert aux vents, celle du Havre en caisses dans des locaux eux aussi ouverts ; pour les autres : expositions immuables et fossilisées dans leurs vitrines des années 1930 ;

3 - rares mais courageux projets muséologiques (Angoulême) et début de la réorganisation des réserves dédiées comme celles du musée d'Aquitaine à Bordeaux en 1987, du muséum de Grenoble en 1986-1989 et du musée national des Arts d'Afrique et d'Océanie de Paris à partir de 1982 ;

4 - très rares inventaires, rareté en grande partie liée à l'absence de spécialistes ;

5 - premières interpellations des communautés concernées par ces collections.

6 En 1981, le monde kanak avait déjà posé les trois questions qui concernaient son patrimoine "dispersé", selon les paroles de Jean-Marie Tjibaou, mais qui devaient, peu à peu, concerner tout le monde :

7 - où sont les objets?

8 - comment sont-ils conservés?

9 - quel discours tient-on sur nous kanak à travers eux ?

10 Ainsi inventaires, conditions de conservation, muséologie, ces trois piliers du métier des musées allaient ces dernières décennies s'appliquer à ce type de collection et ainsi transformer la situation. Une des conséquences sera que les communautés rendues plus informées de la présence de ce patrimoine se poseront comme "experts" de leurs propres affaires (Grognet, F. Du sens perdu de l'autre et du semblable, revue L'Homme, n¹85-186, 2008). Dans l'exposition Collecteurs d'âmes (Rennes, 1986), François Coulon résume en une phrase la situation : "or, bien que présent, tant dans les discours anthropologiques ou esthétiques concernant les objets que dans les galeries qui les exposent, autrui reste invariablement muet faute d'être reconnu et invité".

11 À la suite de l'exposition De Jade et de Nacre, Patrimoine kanak (1990-1991, Nouméa et Paris) qui tenta de prendre en compte ces interrogations nouvelles, une réponse du même ordre a été proposée en 2009 par le musée de Boulogne-sur-Mer par Anne-Claire Laronde et Cécile Ramio autour de la collection Sugpiaq. Cette exposition fut considérée comme un modèle de partenariat entre musées occidentaux et peuples d'origine. Le musée des Beaux-Arts de Rochefort a entrepris depuis quelques années un partenariat par convention avec le Centre Tjibaou.

12 Le muséum d'Histoire naturelle de Rouen, malgré des moyens forts limités, a mis en place une politique d'échanges soutenus avec les maori suite à l'initiative de Sébastien Minchin de restituer la tête tatouée (mokoii) qui fit grand bruit en son temps (2011).

\section{Où sont les objets ? Les inventaires}

13 En 1990 je prenais pour exemple le musée de Boulogne-sur-Mer dont je venais d'inventorier les importantes collections océaniennes. Je concluais sur l'urgence des inventaires spécialisés cherchant à dépasser la simple comptabilité, quand elle existait. Eux seuls permettraient l'élaboration d'un discours nouveau sur ces collections car les inventaires étaient rares. Marie-Charlotte Laroche (dans les années 1960) pour le musée de l'Homme et Anne Lavondès pour l'ORSTOM (Office de la recherche scientifique et 
technique outre-mer), Sylviane Jacquemin et moi-même avions pu en effectuer quelquesuns et réaliser des repérages afin de localiser les fonds existants. Les collections d'Amérique étaient en cours d'inventaire par Pascal Mongne et les fonds Africains bénéficiaient des efforts d'Étienne Féau pour les collections du Sud-Ouest.

Une des difficultés du travail résidait dans la rareté des compétences. Un effort de formation significatif a été engagé depuis par l'École du Louvre qui abandonna le regroupement de tous les arts extra-européens sous l'unique enseigne des Arts primitifs et proposa un enseignement partagé entre Arts d'Océanie et Arts d'Afrique. Il fut complété, à l'université, par les enseignements de Jean-Louis Paudrat, Jean Polet et Louis Perrois.

Une nouvelle génération de conservateurs spécialisés apparaissait ainsi et rendait possible l'exploitation muséologique des inventaires.

Ainsi, en 1996, l'association des conservateurs de Bretagne et celle des conservateurs du Nord-Pas de Calais effectuaient le repérage des collections régionales jetant ainsi les fondements d'inventaires exhaustifs.

17 L'exposition Curieux, Navigateurs et Savants en 1997 qui concluait l'inventaire exhaustif des collections océaniennes du Nord-Pas de Calais rendit visible la richesse de ces collections, et pour certaines l'assurance de leur sauvetage et introduisait une réflexion nouvelle sur ce que l'on pouvait en faire.

Plus récemment, et de manière exemplaire, après trois années de travail d'inventaire raisonné (2011-2014) en France et en Europe "L'Inventaire du patrimoine kanak dispersé" (IPKD) permet de faire un bilan des apports scientifiques qu'une opération bénéficiant de moyens mis à sa disposition par le gouvernement de Nouvelle Calédonie apporte. Avec ses 70 musées visités, près de 15000 objets examinés et de 4000 fiches approfondies examinées, elle a permis d'élargir de manière très significative les corpus aboutissant à une réflexion nouvelle sur des œuvres et des objets pourtant connus de longue date mais dont le faible nombre de spécimens répertoriés ne permettait pas l'étude. Nous avons pu ainsi proposer des interprétations nouvelles concernant la sculpture monumentale kanak, mieux comprendre et réintégrer des objets considérés jusque-là comme marginaux, voire comme des "curios" (objet étrange et intéressant qui évoque la curiosité), dans une catégorie plus large, renouveler l'interprétation des bambous gravés en passant d'une soixantaine de pièces étudiées à près de deux cents et d'asseoir définitivement quelques hypothèses émises par nos prédécesseurs concernant les typologies.

\section{Qu'en fait-on ? L'exposition, la conservation}

Depuis 1990 nous sommes rentrés dans "le temps de la reconnaissance" pour reprendre l'expression de Marine Degli et Marie Mauzé qui la situait en 2006 à l'ouverture du musée du Quai Branly.

20 La naissance du Quai Branly marque avec évidence un tournant mais rendons tout de même hommage aux précurseurs : au musée national des Arts d'Afrique et d'Océanie et à ses expositions comme De Jade et de nacre (1991), Vanuatu (1995) et Les vallées du Niger (1997) ainsi qu'aux musées d'Angoulême depuis 1990 et de Marseille depuis 1992. 
21 Le socle même du musée étant l'exposition permanente, constatons avec satisfaction que depuis 10 ans les rénovations et créations se sont succédées : musée Hebre de SaintClément à Rochefort en 2006, muséum d'Histoire naturelle de La Rochelle en 2007, musée de Boulogne-sur-Mer en 2007, et musée d'Angoulême en 2008. Toutes ces néo-ouvertures ont été l'occasion d'inventaires complets s'appuyant sur ce qui avait été entrepris auparavant.

22 Ce qui s'est considérablement amélioré est l'excellence des conditions de conservation. Partout on rivalise de stockages à haut niveau technique, et d'une surveillance insistante des objets. Notre rôle de conservateur de ce patrimoine est, depuis trente ans, enfin rempli.

\section{Que dit-on de nous ? Réflexion sur le regard}

Vingt années au contact avec le public du musée national des Arts d'Afrique et d'Océanie m'ont montré qu'on ne pouvait continuer, quelle que soit la valeur des textes accompagnant les objets, à laisser penser que les grands tambours dressés du Vanuatu étaient d'authentiques "poteaux de torture". L'imagerie la plus fleurie se manifestait dans les questions et les réflexions des visiteurs pour peu qu'on les rencontrât. Quelques voix chez les anthropologues comme Pascal Dibie et son ouvrage La passion du regard (1998), Sally Price avec son Arts primitifs, regards civilisés (1989), m'avaient convaincu que rien ne permettrait un "dialogue" de culture comme l'annonce le musée du Quai Branly si toute la problématique du regard, de son histoire, des stéréotypes et de son actualité n'était pas prise en charge.

Ce travail sur le regard a fait l'objet de quelques expositions remarquables comme Mythos tahiti Sudsee à Stuttgart en 1987, Le Musée Cannibale de Jacques Hainard à Neuchâtel et Derrière les images au musée d'Aquitaine en 2000. Je réalisai Kannibal et Vahinés en 2001 à Nouméa, au musée national des Arts d'Afrique et d'Océanie et à Chartres. Elle fut suivie en 2002, à Sète, de Paradirama au musée des Arts Modestes avec Cyril Lefebvre, de l'exposition de François Coulon autour du thème des cabinets de curiosités Collecteurs d'âmes en 2006 à Rennes, de L'Autre, les naturels vus par l'Occident au muséum d'Histoire naturelle du Havre en 2008 et plus récemment en 2011, autour de l'œuvre de Victor Segalen, de Rencontres en Polynésie à l'abbaye de Daoulas.

Désormais cette dimension singulière à notre domaine est prise en compte régulièrement au musée du Quai Branly par des expositions temporaires. Son exposition d'ouverture, conçue par Yves Le Fur, D'un regard l'Autre fut de ce point de vue un puissant manifeste complété au fil de la programmation par L'aristocrate et ses Kannibals, Tarzan, Tiki pop. Tout récemment, avec Emmanuel Kasarherou, nous proposions dans l'exposition Kanak, l'art est une Parole, un parcours rapprochant la conception kanak du monde et le regard qu'avait porté l'européen sur cette même société.

Il me semble que la plus aboutie des muséologies en ce domaine est celle qu'a développée en 2010 le musée d'Ethnographie de Cologne dans son exposition permanente. Un effort considérable a été fourni pour faire de ce musée un musée d'ethnographie de notre temps : déconstruction des clichés, réévaluation des collections coloniales et de leur rapport historique aux sciences de l'homme. À l'heure où paraîtra cet article, Barcelone découvrira son nouveau museu de cultures del Mon qui montre les objets et les œuvres d'art pour ce qu'elles sont et de quels peuples elles sont les témoins : objectifs simples 
mais qui ne semblent pas suffisants pour certaines des dernières muséographies proposées.

\section{Actualité des musées : où sont passés les peuples}

Les rénovations et la création récente de quelques grandes institutions européennes brouillent le paysage muséal par les orientations contrastées qu'elles manifestent. Elles montrent, avec obstination, que la question posée en 1990 est toujours en plein débat. Si on s'en tient au problème posé, il y a lieu de constater l'existence de trois points de vue selon la place qu'ils donnent dans leurs expositions permanentes à la rencontre avec d'autres cultures à travers les objets que l'histoire leur a confiés.

\section{Autrui reste muet}

Bâle : le museum der Kulturen qui conserve une des plus importantes collections d'Europe, rouvre en 2012. Le magazine Télérama titre : "Le musée des cultures fait sa révolution par le vide". En effet pratiquement toutes les collections d'ethnologie ont été retirées ou très chichement instrumentalisées pour des démonstrations, illustrant quelques thèmes comme : "Savoir", "Capacité d'agir"... Toutefois entre les lignes on peut lire une interrogation sur l'histoire du regard associée à celle des collectionneurs (ce qui n'est pas tout à fait celle des collections).

\section{Autrui parle d'autre chose}

Deux grands muséums à Toulouse et à Lyon, récemment bénéficiaires de projets ambitieux offrent au public une bonne raison de pousser la porte. Mais ils n'ont pas trouvé, de mon point de vue, la manière d'utiliser les collections que nous a laissées le XIXe siècle.

Dans les deux cas la tentative de parler du "vivant et de l'homme dans l'univers" est tellement ambitieuse que les objets sont noyés, ravalés à la simple fonction d'illustration d'un propos qui les dépasse. On atteint là la limite de la négation du sens de l'objet. $\mathrm{Au}$ point que certains commentateurs plus sévères n'y voient qu'un inventaire à la Prévert ( Télérama). Ces muséographies qui se rapprochent du "concept store" font certes modernes et peuvent attirer le client mais ne permettent guère de faire partager la connaissance d'une autre culture ramassée en un unique et équivoque "théâtre des hommes".

31 Rien dans le parcours qui ne réponde à la question de l'origine de ces objets dans les collections du musée : deux musées coloniaux et un musée missionnaire. On parle des "origines" mais pas de celles des objets. Pas de perspective historique : les objets d'il y a un siècle et demi sont insérés, ici et là, laissant penser que les peuples en question fonctionnent encore à la magie, à la guerre au casse tête tandis que la civilisation occidentale invente, crée et offre à l'humanité la cocotte minute et le microscope (pas de religions, pas d'attitude envers la mort, pas de mythes fondateurs pour nous?).

Pourtant j'avais été très enthousiasmé en voyant ce musée sous l'impulsion de Michel Côté enrichir ses collections par des œuvres contemporaines qui auraient pu montrer l'heureuse persistance de la diversité humaine et de son génie artistique. 
La mise en rapport abrupte d'un silex avec un masque africain ne peut aboutir, sauf travail sur le regard, particulièrement absent, qu'à des rapprochements déclencheurs de stéréotypes, d'imageries hasardeuses. De la même manière, est-ce qu'expliquer la structuration des sociétés par une collection d'armes du XIXe siècle est si éclairant ? Où veut-on en venir?

\section{Autrui parle de lui et de lui-même}

Par contre à Genève, "Archives de la diversité humaine", un des thèmes développés par le nouveau musée d'Ethnographie exploite au mieux ses collections et rejoint les réalisations de Paris, Cologne et Barcelone qui tentent de donner aux collections des siècles derniers un statut qui interroge le public sur la diversité humaine.

Une remarque enfin sur une "idée" fort partagée en ce moment : le recours assez systématique au "cabinet de curiosités" montre que le procédé est bien commode justifiant le fourre-tout et que c'est manifestement méconnaitre l'esprit de ce que furent ces cabinets de curiosités de les traiter ainsi. On utilise ainsi plus facilement le stock d'objets, il est vrai souvent disparate, conservés dans nos musées, mais on évite la réflexion et le travail vis-à-vis du public et des communautés concernées.

\section{Conclusion}

La disparition des musées d'ethnographie au profit d'une muséologie de l'Universel, applicable à toutes les sociétés de la même manière que la mondialisation, entérine la fin des peuples et de la diversité des sociétés et des espèces.

Est-ce que certaines des réalisations commentées ci-dessus ne justifieront pas les critiques qui furent exprimées au siècle dernier envers les expositions dites de "comparaison" pour lesquelles ont été rassemblées une grande partie de ces collections ? Dans les cas évoqués, l'objet a tendance à être réduit à un instrument d'un discours qui lui est étranger, borné à sa capacité d'illustration.

Face à cette nouvelle situation, il ne serait pas étonnant que les peuples d'origine de ces collections s'interrogent sur l'utilisation qui est faite de leur patrimoine. Y porter attention ne devrait pas laisser penser à certains qu'il s'agit d'un dolorisme issu d'une culpabilité coloniale mal assumée. Cela ne changerait rien à l'existence des sollicitations le plus souvent passionnantes par les collaborations et les échanges qu'elles induisent entre institutions et musées. D'autant que la tendance observée au cours de ces toutes dernières années est plutôt au retrait de ces collections qui s'ajoute déjà au faible volume exposé (en France moins de $10 \%$ ), pourrait laisser penser que ces collections sont désormais, certes excellemment conservées, mais vacantes. Les opérations de dépôts de longue durée mise en œuvre avec la Nouvelle Calédonie depuis 20 ans sont l'exemple d'une relation réussie et d'une manière nouvelle de considérer ces objets et ces œuvres d'art. 


\section{RÉSUMÉS}

À l'occasion des 30 ans de l'OCIM, La Lettre de l'OCIM propose à ses lecteurs, dans chaque numéro de l'année 2015, un retour sur un article marquant de l'histoire de la revue. À partir d'un corpus d'articles choisis par la rédaction de La Lettre de l'OcıM, les membres du comité des Publications de l'OCIM ont sélectionné plusieurs contributions.

Dans cette perspective, il a été demandé à l'auteur ou à un expert du domaine de revisiter la problématique exposée dans l'article à la lueur des changements intervenus - notamment dans les pratiques professionnelles - depuis son écriture et de proposer des éléments prospectifs sur la question.

Roger Boulay revient sur son article "Actualité d'un débat qui s'éternise : que faire des collections d'objets exotiques ?" publié dans le ${ }^{\circ} 9$ de La Lettre de l'OCIM de mai-juin 1990 et dans lequel il constatait les hésitations et la confusion entourant alors les projets muséographiques liés à ces collections : il livre son point de vue de scientifique de terrain, travaillant avec les communautés, sur les évolutions et réalisation actuelles.

\section{INDEX}

Mots-clés : collection, objet exotique

\section{AUTEUR}

\section{ROGER BOULAY}

anthropologue, muséologue et spécialiste des Arts d'Océanie

roger.boulay@mncparis.fr 